\title{
Mundos virtuales y el aprendizaje inmersivo en educación superior
}

\section{Virtual Worlds and Immersive Learning in Higher Education}

\author{
Rocío Janett Ayala Pezzutti \\ Universidad César Vallejo, Lima, Perú \\ ORCID: https://orcid.org/0000-0001-7930-0644 \\ Carlos Miguel Laurente Cárdenas \\ Universidad César Vallejo, Lima, Perú \\ ORCID: https://orcid.org/0000-0003-0566-4992 \\ César Daniel Escuza Mesías \\ Universidad César Vallejo, Lima, Perú \\ ORCID: https://orcid.org/0000-0003-2150-1173 \\ Luis Alberto Núñez Lira* \\ Universidad César Vallejo, Lima, Perú \\ ORCID: https://orcid.org/0000-0003-3542-9117

\section{Jorge Rafael Díaz Dumont} \\ Universidad Nacional Autónoma de Tayacaja Daniel Hernández Morillo, Huancavelica, Perú \\ ORCID: https://orcid.org/0000-0003-0921-338X
}

\section{Recibido 16-04-19 Revisado 30-05-19 Aprobado 04-11-19 En línea 07-01-20}

\section{*Correspondencia}

Email: lnunezl@ucv.edu.pe

\section{Citar como:}




\section{Resumen}

La sociedad actual se ha convertido en un oscilar constante de cambios en cada aspecto de la vida; académico, económico, cultural, político, entre otros. Los cambios se han generado con la inserción de tecnología en las actividades diarias, donde el aprendizaje no queda exento a estos cambios. Según Negroponte (1995), cuando la ciencia se une con la tecnología esta produce cambios significativos en nuestra vida, así como la forma como entendemos la realidad. Hoy la sociedad del conocimiento está potenciada por la tecnología y por la creación de nuevas y diversas formas de interactuar como personas; los mundos virtuales que son una representación gráfica de la realidad diseñada en $3 \mathrm{D}$, es una de ellas. Second Life es uno de estos mundos virtuales, convirtiéndose de un sitio de mero entretenimiento y vida social en la web, a establecerse en ambiente que fomenta la creatividad, el trabajo colaborativo, la visión empresarial, el desarrollo de habilidades tecnológicas, el desarrollo de experiencias de aprendizaje en entornos 3D, entre otros aspectos. Asimismo, el aprendizaje inmersivo posibilita la interacción en ambientes tridimensionales. Allí las actividades se realizan dentro de un mundo construido artificialmente el cual asemeja la realidad, esto permite construir el aprendizaje a través de las experiencias propias que tenga la persona en interacción con el entorno y las experiencias entre los usuarios de este mundo virtual. Por tal razón, se propone el uso del mundo virtual Second Life como una herramienta formativa en el aprendizaje inmersivo.

Palabras Clave: Aprendizaje en línea; Internet; Second Life; Espacio abierto.

\section{Summary}

Today's society has become a constant swing of changes in every aspect of life; academic, economic, cultural, political, among others. The changes have been generated with the insertion of technology in daily activities, where learning is not exempt from these changes. According to Negroponte (1995), when science meets technology, it produces significant changes in our lives, as well as the way we understand reality. Today the knowledge society is empowered by technology and by the creation of new and diverse ways of interacting as people; the virtual worlds that are a graphic representation of the reality designed in 3D, is one of them. Second Life is one of these virtual worlds, becoming a site of mere entertainment and social life on the web, to be established in an environment that fosters creativity, collaborative work, business vision, the development of technological skills, the development of experiences of learning in 3D environments, among other aspects. Likewise, immersive learning enables interaction in three-dimensional environments. There the activities are carried out within an artificially constructed world which resembles reality, this allows us to build learning through the person's own experiences in interaction with the environment and the experiences among the users of this virtual world. For this reason, the use of the Second World virtual world is proposed as a training tool in immersive learning.

Keywords: Electronic Learning; Internet; Second Life; Open Spaces.

\section{Introducción}

La sociedad del conocimiento es potenciada fundamentalmente por la tecnología y el incremento de los mundos virtuales, posibilitando la creación de múltiples y novedosas maneras de relacionarse entre personas, lo que poco a poco estaría abriendo el paso a nuevas maneras de vivir y educarse. Cualquier tecnología unida a la ciencia está produciendo cambios significativos en nuestra forma de vida y en el entendimiento que poseemos de la realidad (Negroponte 1995). 
Las instituciones educativas alrededor del mundo han ido incorporando a sus prácticas pedagógicas el uso de entornos simulados por computador cuyos lugares y objetos son representados de manera gráfica en 3D. Second Life es un digno representante de lo ya mencionado; es un espacio creado y evolucionado por sus propios usuarios, un mundo inhabitado en tres dimensiones que reúne a individuos para compartir experiencias de un modo revolucionario, en donde todos son capaces de construir a su alrededor convirtiéndose en seres demiurgos (Bulkley, 2007).

Poco a poco este mundo virtual pasó de ser un sitio de entretenimiento y vida social a ser un ambiente que promueve la creatividad, visión empresarial y el desarrollo de experiencias de enseñanzas interactivas en 3D; en otras palabras, en este último campo, Second Life ofrece soluciones de educación virtual para mejorar un plan de estudios existente o crear nuevos modelos para el aprendizaje colaborativo, comprometido e inmersivo. Asimismo, cuando hablamos de un aprendizaje a través de tecnologías de la web nos referiremos al aprendizaje inmersivo que es el que se da por medio de entornos tridimensionales, esto a su vez proporciona a los educadores la capacidad de conectarse, comunicarse y colaborar de una manera que realce en gran medida la experiencia de aprendizaje (Martínez 2014).

El aprendizaje inmersivo basado en el soporte de las tecnologías de la información, las redes sociales y la ludificación se da gracias a los avances tecnológicos relacionados con procesos que son ricos en interfaces de usuario; los cuales representan situaciones realistas en donde podemos lograr procesos pedagógicos complejos ya que son entornos en los cuales los estudiantes pueden participar y sumergirse en experiencias que fomenten un aprendizaje significativo (Ly, Saadé \& Morin, 2017).

El aprendizaje inmersivo consta de actividades que logra que los usuarios se sumerjan en un mundo construido artificialmente (mundo virtual) el cual se asemeja a la realidad. Aquí, las interacciones de los usuarios con elementos como personas, objetos, actividades, búsquedas y otros artefactos simulados otorgan una oportunidad que podría ser difícil de crear en el mundo real debido a riesgos y/o gastos que puede ocasionar (Inks \& Ávila, 2008).

Por ejemplo, el Instituto Nacional de Salud de Estados Unidos (NIH) posee un sitio que simula un pueblo en el cual se le permite al usuario conocer los químicos tóxicos en el suelo, agua y aire que pueden ser hallados en lugares como fábricas, centros de salud, colegios, casas, parques y puertos pesqueros de zonas urbanas o rurales. Esta experiencia logra en los estudiantes una visión memorable y esclarecedora del tema, que en muchos casos el aula tradicional no puede ofrecer; además, estas actividades son muy gratificantes y atractivas para estudiantes de todas las edades.

En el caso de Latinoamérica son principiantes las investigaciones y aplicaciones del aprendizaje inmersivo y los mundos virtuales en educación, más específicamente de Second Life, esto comparándolo con países que vienen implementando trabajos y proyectos educativos desde hace ya varios años. Los países latinos con mayor presencia en este metaverso son México, Colombia, Venezuela, Perú y Brasil.

Hoy por hoy, en el Perú los temas de aprendizaje inmersivo y los mundos virtuales no son ampliamente conocidos pues existen muy pocas investigaciones realizadas. Sobre este último, existen instituciones de nivel superior como la Pontificia Universidad Católica del Perú y la Universidad San Martin de Porres que trabajan con este mundo virtual y hasta tienen construcciones en él. De las cuales, en particular la Pontificia Universidad Católica del Perú es una de las más involucradas en Second Life en todo Latinoamérica (Elías \& Evaristo 2009).

Las investigaciones que han precedido nos muestran resultados relevantes que serán de utilidad en esta investigación; así se reconoce que los entornos inmersivos otorgan herramientas 
valiosas para mejorar la teoría y la práctica. Es por ello que se debe proporcionar entornos de aprendizaje inmersivos en los que los alumnos puedan presentarse, encarnarse y participar activamente potenciando y facilitando el aprendizaje de un modo transformacional. (Cheney y Terry, 2018).

Las oportunidades pedagógicas que otorgan no se circunscriben al conocimiento teórico implantado en cursos tradicionales, sino que abarcan habilidades clave que son transversales y no disciplinares. Así, combinando métodos de enseñanza constructivista y la efectividad de los entornos 3D se logra el florecimiento de capacidades y habilidades intercurriculares, siendo este proceso más intrínsecamente motivador para algunos estudiantes (Abbott, Jeffrey, Gouseti, Burden, y Maxwell, 2017).

En cuanto a la práctica, un modo transformador y que se ha ido incrementando con el correr de los años es el juego. Con relación a ello se pueden utilizar entornos de aprendizaje inmersivos lúdicos que logran el aumento de la curiosidad y estimulan el aprendizaje al envolver al usuario en mundos virtuales interesantes que se asemejan a hechos reales, eventos, etc. Haciendo uso de un avatar, la simulación y la virtualización de los entornos de aprendizaje inmersivo en 3D, combinándolo con una narración atrayente y el aprendizaje que se basa en juegos, se logra una narrativa denominada "gamificada" el cual permite que el aprendizaje se enfoque en el problema, se ajuste y sea generado por el contexto. Estudiosos afirman que los logros de aprendizaje, conocimiento procedimental y declarativo, y habilidades del pensamiento de orden superior son enriquecidas al añadirle una dosis de gamificación (Mystakidis \& Berki, 2018; Kim, Song, Lockee \& Burton, 2018).

Las tecnologías de la información y su uso en todo el mundo impactan las formas de aprendizaje de los estudiantes y aquellos modelos pedagógicos que se les asocian. Enseñar y aprender ya no es lo mismo en la actualidad y el cambio de paradigma aún no ha sido resuelto. Es necesario desarrollar una metodología eficaz en el proceso de enseñanza-aprendizaje, el cual permita comprender cómo se crea el conocimiento en diferentes disciplinas profesionales.

El conocimiento profundo de los métodos de aprendizaje inmersivo fomenta la aplicación del aprendizaje inmersivo experiencial el cual permite incrementar en los estudiantes su rendimiento y compromiso; además, un gran porcentaje de las habilidades requeridas en los trabajadores de hoy es el denominado experiencial. Por todo esto, los educadores deben comenzar a avanzar hacia modos más auténticos de educación los cuales pueden lograrse a través de este tipo de aprendizaje.

El problema es que impartirlo no es fácil, es necesario proporcionar experiencias auténticas que simulen la vida real; esto puede llevar mucho más tiempo de planificación, la búsqueda de nuevos modos de evaluar y hasta ser más costoso. A pesar de ello, para un número creciente de educadores, las simulaciones de aprendizaje inmersivo se han convertido en una manera más llamativa de proporcionar auténticas oportunidades de aprendizaje experiencial que son atractivas y escalables (Ly, Saadé \& Morin, 2017; Beckem \& Watkins, 2012).

La sociedad actual exige profesionales competentes y la educación superior debe estar a la altura de estas exigencias, en donde los estudiantes interactúen en entornos virtuales y a su vez potencien capacidades y destrezas que dentro de nuestra sociedad actual se establecen como necesarias en cada profesional. A futuro tendremos que cerrar la brecha entre la manera en que los estudiantes interactúan y construyen conocimiento en un entorno rico en medios, y cómo ayuda el ambiente de aula. Esto necesitará un cambio a entornos inmersivos en donde se preste mucha atención a las bases de conocimiento y los recursos que los estudiantes manejan y traen al aula. Para ello, los docentes deberán utilizar enfoques pedagógicos adaptativos que logren una comprensión matizada de 
la orientación epistemológica, el lenguaje y la naturaleza de los entornos pro sociales (Nuñez \& Duarte, 2017; Hand, Cavagnetto, Chen \& Park, 2016).

Adicionalmente se encontraron investigaciones que anteceden y aportan al presente trabajo de investigación en torno a los mundos virtuales. Es en este sentido que se necesita la comprensión de las oportunidades, fortalezas y desafíos que conlleva la tarea de enseñar dentro de estos entornos y utilizar este espacio como herramienta educativa.

En cuanto a las fortalezas, se encuentra la posibilidad de integrar los MV como recursos didácticos. También está el hecho de ser novedosos espacios de aprendizaje que favorecen de múltiples maneras la construcción del conocimiento colectivo y el trabajo colaborativo. Asimismo, nos abren nuevas rutas de investigación para el análisis de la educación del siglo XXI (Ángel, Valdés \& Guzmán, 2017; Quinche \& González, 2011; Márquez, 2010).

En las oportunidades, la posibilidad de desarrollar distintos modos de acceso a la información y conocimiento teniendo como base los principios lúdicos, así como de favorecer novedosos medios de comunicación que a su vez se vuelve parte de un riesgo que enfrentan al interactuar en los mundos virtuales (Ángel, Valdés \& Guzmán, 2017; Gabarda, Orellana \& Pérez, 2017).

Los desafíos indican que el trabajo como docente dentro de estos espacios requiere diversos retos, tales como definir los contenidos temáticos, proponer una estrategia didáctica, aprender y dominar el uso de los mundos virtuales (Ángel, Valdés y Guzmán, 2017). También, es necesario construir redes de convivencia que propicien la responsabilidad ciudadana en donde todos los involucrados aprendan a respetarse obedeciendo normas dentro de la red (ciber convivencia), asegurando así un ambiente de seguridad y respeto para todos en el mundo virtual. Recordando que los usuarios interactúan dentro del contexto virtual en donde se establecen relaciones y realidades simbólicas, generando a través de ellas una nueva vida; la cual es paralela a la vida concreta, y que amplía el panorama de espacio social real a la que estaba acostumbrado (Gabarda, Orellana \& Pérez, 2017; Paz, 2017).

Por último, entre las amenazas se encuentra la limitación en los requerimientos de infraestructura tecnológica. Los aspectos técnicos deben ofrecer la interfaz apropiada, con la finalidad que exista funcionalidad en el uso de aulas virtuales pues los problemas de acceso o retardo en la comunicación puede provocar desmotivación entre los participantes de las comunidades virtuales. Asimismo, las instituciones educativas necesitan un proyecto que promueva la sensibilización para el uso pertinente y saludable de la web y de las interacciones que se hagan a través de esta. Una educación que se basa en la restricción y el control del uso de tecnologías no tiene sentido; en cambio, es más efectivo una educación a través del cual aprendan a usar estas tecnologías de manera responsable y crítica para poder así enfrentar las amenazas que encuentren durante su uso (Ángel, Valdés \& Guzmán, 2017; Buitrago, 2016).

La literatura revisada nos habla que el mundo de la educación ha sido afectado desde hace muchos años por la intrusión de manera intensa de la tecnología, favoreciendo el crecimiento de entornos de teleformación para el aprendizaje inmersivo, en los cuales los campus físicos de las instituciones de nivel superior se están virtualizando, ofreciéndose nuevos escenarios de aprendizaje en el cual el profesor tomando la forma de avatar se convierte en guía en el acceso a la información y no un simple transmisor de la misma (Joyanes, 2017).

Un mundo virtual es una representación por computadora de un espacio, generalmente es la proyección en 3D de unidades geomorfológicas, ciudades, objetos, animales, personas, etc.; que han 
sido digitalizados. Aquí nos movemos y socializamos con los demás usuarios a través de una imagen que nos representa, denominada avatar. (Grané, Frigola \& Muras, 2007).

Estos mundos otorgan modernos modos de comunicación y manejo de recursos en el ciberespacio proporcionando a los usuarios, o alter egos virtuales, una gran variedad de oportunidades de intercambio, cooperación e interlocución de modos diversos con los demás participantes inmersos en estas plataformas y con el mismo entorno virtual propiamente dicho. De esta manera ellos experimentan la sensación de "estar en" los distintos eventos y situaciones que ocurren. Todo esto permite que los mundos virtuales se conviertan en un lugar ideal para realizar actividades de aprendizaje por medio de las distintas representaciones de la realidad (Cruz, Therón, Pizarro \& García-Peñalvo, 2013). Es decir, los mundos virtuales nos proporcionan un entorno inmersivo en donde la sensación de "estar dentro" es una de las características que lo hace ideal para ser usadas en labores pedagógicas.

Los pros que la tecnología ofrece a través de este tipo de recursos son numerosos, entre ellos se encuentra el poder visitar o reproducir lugares en 3D a los cuales sería difícil acceder en centros de enseñanza - aprendizaje del nivel básico y superior que no cuentan con los recursos necesarios para realizar construcciones físicas o viajes en el mundo real; además, la asistencia de los usuarios no tiene barreras de ubicación o lugar del cual provienen. Es por todo ello que se vuelve importante su uso y profunda comprensión porque a través de esto se logra la mejora y amplitud de sus aplicaciones en la pedagogía (García-Peñalvo, Cruz-Benito \& Therón, 2014).

Por su parte, Martínez (2014), investigadora Jr. del Centro de Investigación Científica y Educativa de Samara National Research University (Rusia), Doctora en Tecnología Educativa en la Universidad de Sevilla (España), quien ha participado y coordinado proyectos de e-learning a nivel internacional y ha publicado tres libros sobre el uso de las TIC en el aprendizaje relacionados con entornos virtuales 3D, mundos virtuales y SLoodle, añade que en los mundos virtuales nuestros estudiantes son el elemento más céntrico de todo el proceso de instrucción, por medio de crear y diseñar experiencias educativas claras y concisas para ellos, dentro de los cuales se logre enfocar y abordar el aprendizaje de un modo más práctico. De esta manera se inician diversas situaciones para la producción de experiencias educativas reales, acomodadas, compartidas y sobre todo que otorgan un entorno que cambia las reglas que definen el éxito de los estudiantes y los sistemas de valoración tan juzgados actualmente. Existen una gran variedad de mundos virtuales usados en educación como lo son Simschool, MinecraftEDU, Small Worlds, ScienceSim, Active Worlds Educational Universe, Secret Builders, WizWorld Online, WoWinSchool, etc.

En la presente investigación nos enfocaremos solo en Second Life, mundo virtual en 3D creado por la empresa americana Linden Lab en el año 2002, el cual es uno de los más concurridos a nivel mundial y que inicialmente fue creada con el fin de que sus usuarios se entretengan y socialicen para posteriormente ser usada también en el rubro educativo. Cuando visitas su página puedes conocer más sobre las bondades de este mundo virtual. Lo primero que encontramos es que se autodenomina una potente plataforma para la creatividad en donde se pueden encontrar objetos 3D interactivos, pasar experiencias únicas y vivir en comunidades globales las cuales son creadas por gente como nosotros. Menciona que podemos convertirnos en comerciantes creando y vendiendo artículos virtuales en un mercado global pues Second Life ofrece infinitas oportunidades para los diseñadores 3D, modeladores, scripters, etc., para beneficiarse de su creatividad. Podemos, además, convertirnos en propietarios y que el alquiler de tierra virtual a los inquilinos puede ser un negocio muy lucrativo mostrando como ejemplo un propietario de Second Life que fue incluido incluso en la portada de Business Week de la revista Time como "el primer millonario de Second Life". 
Nos ofrece también la posibilidad de crear experiencias como el desarrollar nuestros propios juegos de inmersión, lugares de música, clubes de baile, espacios de entretenimiento para adultos o cualquier experiencia virtual que se pueda imaginar. Se puede ganar Linden Dollars (que pueden ser cambiado a dólares americanos reales) trabajando a tiempo completo o parcial para un negocio en este mundo virtual. Los clubes que allí se han creado necesitan anfitriones, DJs y otros. Las tiendas a menudo necesitan vendedores y personal de apoyo al cliente. Second Life nos invita a conectarnos con una comunidad mundial conociendo a gente real de todo el mundo y chateando con ellos. Finalmente, nos invita a crear sugestivas experiencias de enseñanza interactiva en 3D ofreciendo a las organizaciones que reúnan las condiciones un $50 \%$ de descuento al comprar terrenos virtuales fomentando con ello el incremento del uso de este mundo virtual en educación.

Diversos autores han explorado el uso de este mundo virtual en el proceso de enseñanza aprendizaje. Dentro del ambiente educacional, Second Life muestra mayor envergadura debido a sus particulares tales como la interacción, la posibilidad de tener un cuerpo a través de avatares y la perseverancia. La participación del alumno es totalmente activa iniciándose desde que tiene libertad de crearse a sí mismo, de comunicarse y socializar no solo con sus pares sino con personas de todo el mundo, de construcción de su entorno virtual tales como laboratorios, aulas, edificios y cualquier espacio o material educativo en general que les permita recrearse y el desarrollo y aplicación de distintas estrategias educacionales (Díaz, 2016).

Las ventajas que posee Second Life son múltiples ya que posibilita la interacción entre avatares que copian el comportamiento humano como conversar, danzar, cambiar la vestimenta y hasta realizar intimidad entre ellos. Además, información puede ser entregada y recibida, documentos pueden ser leídos y compartidos, videos pueden ser vistos, audios escuchados, representaciones de lugares reales e imaginarios pueden ser visitados, objetos pueden ser creados manipulados o creados y servicios pueden ser ofrecidos.

Existen cinco maneras de cómo los mundos virtuales pueden ser utilizados: para socialización, pudiendo conectarte con gente real de todas partes del mundo; para entretenimiento, en donde puedes disfrutar de juegos y hasta música asistiendo a conciertos en vivo; para negocios o comercio, creando tu propio mundo mediante la compra o alquiler de terrenos logrando ganar así dinero real; y para formación, creando novedosos modelos de enseñanza participativa y colaborativa en espacios tridimensionales (Adaptado de Martínez, 2014).

En cuanto al aprendizaje inmersivo, una definición muy concreta nos la ofrece Martínez (2014) al mencionar que es aquel que se desarrolla por medio de ambientes tridimensionales. Sabemos que el aprendizaje en sí es una de las funciones de la mente más importantes en los seres humanos, animales y sistemas artificiales. Ahora bien, la predicción de los aprendizajes basados en tecnología es inmersivo. Esto quiere decir que los estudiantes sentirán que están "en" la situación de aprendizaje y están realizando prácticas necesarias para mejorar a través de la toma de decisiones, del mando de un equipo de personas, o quizás del manejo de algún instrumento especializado. Podrán ser entrevistados para obtener una plaza de trabajo o realizar alguna actividad con retroalimentación en tiempo real, ello gracias a que la tecnología facilita que todo ocurra de este modo y desde cualquier lugar. Pero, el aprendizaje inmersivo no se trata solo acerca de la tecnología sino también del plan diseñado para que los estudiantes practiquen en contexto, incrementando conocimientos y mejorando habilidades y competencias. Podremos decir entonces que seleccionar la tecnología será una decisión importante y que actualmente hay muchas de ellas que pueden ser usadas para lograr experiencias inmersivas (Olbrish, 2013).

Hay tres perspectivas principales en las que se utiliza el "aprendizaje inmersivo": en un entorno experiencial, a través de un método constructivista y mediante una colaboración activa o 
social. Las investigaciones relacionan este término con un contexto específico y gracias a la ayuda de las Tics (Ly, Saadé \& Morin 2017).

El aprendizaje inmersivo experiencial se representa por diversas actividades que permiten que los estudiantes se sumerjan en un lugar que se asemeja a la realidad y que es construido de manera artificial (mundo virtual). Aquí podrán aprender a través de experiencias simuladas e interacciones de los estudiantes con componentes como actividades, gente, tareas, cosas, etc., que podrían ser difíciles de crear en el mundo real ya que conllevan grandes gastos o riesgos. Las experiencias inmersivas otorgan a los estudiantes un modo memorable de ver un tema, una visión esclarecedora que un aula tradicional no ofrece. Las experiencias logradas son atractivas y placenteras pues las tecnologías de inmersión en las cuales se incluye la realidad virtual actualmente otorgan, a los centros de estudios y alumnos, el acceso a una plataforma de aprendizaje personalizable y beneficiosa (North, 2014). Además, nos permite el desarrollo de la competitividad éxito de la practica del docente, en la medida que genera valor y desarrollo cognitivo (Núñez, et al, 2019)

Mediante el aprendizaje constructivista cada alumno aprende a su ritmo. Actualmente se pueden crear situaciones en un entorno diseñado a distintos niveles de dificultad considerando los conocimientos previos de los estudiantes y permitiéndoles replantear los conceptos erróneos que tengan de un tema. De esta manera, mediante la reflexión e introspección se logra el procesamiento del conocimiento que se requiere alcanzar y asimilar.

Complementariamente generan el desarrollo de competencias genéricas y específicas, que determinarán el aprendizaje propiamente o dicho o el desarrollo de cualquier actividad, inclusive en las laborales. (Núñez, et al, 2018). El docente trabajará involucrando a los estudiantes en actividades de reflexión a través de la observación y probándolos en una conceptualización abstracta de algún tema puntual, es por ello que el conocimiento trabajado en alguna actividad puede ser orientado o estructurado. Además, se puede monitorear su proceso de aprendizaje y a la vez permitirles se autónomos en ese aspecto pues podrán pasear libremente por el lugar, interactuar con sus pares, obtener información que sea de su interés mientras el maestro recibe información sobre su progreso del aprendizaje consciente e inconsciente (Fernandes, Roja \& Eyre, 2003).

El aprendizaje social o colaborativo, el cual es muy popular actualmente gracias a las redes sociales, posibilita que los estudiantes compartan y aprendan de manera recíproca. La interacción tiene un rol importante dentro de los mundos virtuales ya que estos permiten el aprendizaje en grupo de manera parecida a las experiencias físicas en clase; y aún mejor ya que se crean espacios de trabajo en donde los estudiantes que se encuentren lejos puedan reunirse o conectarse para el intercambio experiencial y cognitivo (Stefan, 2012).

El presente estudio se enmarca en el aprendizaje inmersivo y el uso formativo del mundo virtual Second Life; en consecuencia, la formulación del problema general de investigación responde a la siguiente interrogante: ¿Cómo es el uso del mundo virtual Second Life como herramienta formativa para docentes del nivel superior en el aprendizaje inmersivo - 2020? Del mismo modo, los problemas específicos que se desprenden responden a la interrogante ¿cómo es el uso educativo del mundo virtual Second Life como herramienta formativa para docentes del nivel superior en el aprendizaje inmersivo experimental, constructivista y social o colaborativa - 2020.

Con el propósito de responder a esta pregunta se realiza la exploración del mundo virtual Second Life, en donde participan docentes que lo utilizan para la enseñanza de diversas materias del nivel superior en distintas instituciones alrededor del mundo incluyendo el Perú. De este modo, este metaverso se convierte en un recurso que el profesor emplea para facilitar contenidos, mediar 
experiencias de aprendizaje, provocar encuentros o situaciones con objetivos específicos, desarrollar habilidades cognitivas, enriquecer la valoración del aprendizaje, etc.

Finalmente, el objetivo general está orientado a analizar cómo es el uso del mundo virtual Second Life como herramienta formativa para docentes del nivel superior en el aprendizaje inmersivo - 2020. Los objetivos específicos se relacionan con cada una de las categorías del aprendizaje inmersivo, formulados de la siguiente manera: analizar cómo es el uso del mundo virtual Second Life como herramienta formativa para docentes del nivel superior en el aprendizaje inmersivo experimental, constructivista y social o colaborativa - 2020.

\section{Método}

La perspectiva metodológica que se ha seguido es la cualitativa. Aquí se intenta comprender la experiencia, los factores que intervienen en el hecho a investigar, teniendo en cuenta que la realidad se construye por las personas en interacción con el mundo social que lo rodea. En una investigación cualitativa se fija el interés por comprender los significados que construyen los individuos, en otras palabras, qué sentido le dan al mundo y a las experiencias vividas en este. Se explica, además, que las experiencias de los individuos presentan un significado que es recogido por las percepciones propias del investigador. Por otro lado, se sigue una estrategia de investigación inductiva cuyo producto es ricamente descriptivo. Desde esta perspectiva el investigador es el principal instrumento para obtener y analizar los datos (Hernández, Fernández \& Baptista, 2014).

El diseño de investigación propuesto es el fenomenológico pues su propósito principal es explorar, describir y comprender las experiencias de las personas con respecto a un fenómeno (el metaverso Second Life) y descubrir los elementos en común de aquellas vivencias. Es decir, se pretende describir el comportamiento de los participantes dentro del Second Life, así como dar a conocer qué uso le dan para que pueda convertirse en un recurso didáctico que propicie el aprendizaje inmersivo de sus estudiantes, partiendo de supuestos anticipándose a los resultados de la investigación, construyendo concepto o hipótesis que permitirán desarrollar la investigación empírica más adelante, para la profundización del estudio (Yuni \& Urbano 2005).

Además, nos permitirá el análisis comparativo de los resultados de la investigación, y así, determinar el carácter de la misma en comparación con otros modelos teóricos referidos al tema, para determinar el nivel de avance en el desarrollo de la innovación pedagógica (Popper, 1980).

La población empleada para el estudio fueron estudiantes de una universidad privada del Perú, donde los instrumentos empleados fueron la observación participante, la entrevista a los participantes y expertos, así como el análisis documental recogido en la formulación de las clases ejecutadas.

\section{Resultados}

Los resultados obtenidos fueron luego de sistematizar a través de una matriz categorial, donde resaltan tres categorías clave: aprendizaje inmersivo experiencial, aprendizaje inmersivo constructivista y aprendizaje inmersivo social o colaborativo, subdividiéndose en subcategorías e indicadores para su medición; además los ítems nos permitieron realizar las preguntas a profundidad de los expertos entrevistados (ver tabla 1). 
Tabla 1.

Categorización del aprendizaje inmersivo

\begin{tabular}{|c|c|c|c|}
\hline Categoría & Subcategoría & Indicadores & Ítems \\
\hline \multirow{2}{*}{$\begin{array}{l}\text { Aprendiz } \\
\quad \text { aje } \\
\text { inmersivo } \\
\text { experienc } \\
\quad \text { ial }\end{array}$} & $\begin{array}{l}\text { Interacción } \\
\text { con el } \\
\text { entorno }\end{array}$ & $\begin{array}{lr}\text { - Realiza actividades } \\
\text { experienciales utilizando } \\
\text { el entorno virtual. } \\
\text { - Comunica lo que conoce } \\
\text { utilizandorras distintas } \\
\text { herramientas del } \\
\text { entorno. }\end{array}$ & $\begin{array}{l}\text { - ¿Consideras a Second Life como una } \\
\text { herramienta útil para la enseñanza } \\
\text { experiencial? ¿Por qué? } \\
\text { - ¿Qué actividades de tu especialidad has } \\
\text { realizado en Second Life? } \\
\text { - Dentro de Second Life ¿Qué herramienta } \\
\text { utilizas principalmente para comunicar } \\
\text { ideas? }\end{array}$ \\
\hline & $\begin{array}{l}\text { Manipulació } \\
\text { n del entorno }\end{array}$ & $\begin{array}{l}\text {-Diseña y construye } \\
\text { espacios y objetos } \\
\text { utilizando herramientas } \\
\text { virtuales. } \\
\text { - Evalúa la funcionalidad } \\
\text { de los espacios y objetos } \\
\text { creados. }\end{array}$ & $\begin{array}{l}\text { • ¿Has realizado construcciones con fines } \\
\text { educativos dentro de S L? Menciónalas } \\
\text { y/o descríbelas } \\
\text { •Para la construcción de espacios de } \\
\text { aprendizaje. ¿Qué debemos tener en } \\
\text { cuenta principalmente? } \\
\text { • Según su apreciación. ¿Qué características } \\
\text { debe tener un objeto construido en SL } \\
\text { para lograr el aprendizaje? } \\
\text { •QQué características debe tener un espacio } \\
\text { de aprendizaje en SL para lograr un } \\
\text { aprendizaje vivencial? }\end{array}$ \\
\hline \multirow{3}{*}{$\begin{array}{l}\text { Aprendiz } \\
\text { aje } \\
\text { inmersivo } \\
\text { constructi } \\
\text { vista }\end{array}$} & $\begin{array}{c}\text { Aprendizaje } \\
\text { autodirigido } \\
\text { del usuario }\end{array}$ & $\begin{array}{l}\text {-Planifica actividades, } \\
\text { apoyándose en el } \\
\text { conocimiento de lugares } \\
\text { antes visitados. } \\
\text { - Reflexiona respecto a las } \\
\text { actividades de } \\
\text { aprendizaje diseñadas } \\
\text { dentro del entorno. }\end{array}$ & $\begin{array}{l}\text { - Dentro de Second Life, cuál sería la mejor } \\
\text { decisión, ¿trazar una ruta para que el } \\
\text { estudiante visite determinados lugares o } \\
\text { permitir que libremente haga las visitas? } \\
\text { ¿Por qué? } \\
\text { ¿Qué indicadores te demuestran que una } \\
\text { clase en SL estuvo bien realizada? } \\
\text { •Quién cree que aprende más en una clase } \\
\text { en SL, el docente o el estudiante? }\end{array}$ \\
\hline & $\begin{array}{c}\text { Monitoreo en } \\
\text { el } \\
\text { aprendizaje }\end{array}$ & $\begin{array}{l}\text { - Selecciona espacios } \\
\text { propicios para el } \\
\text { desarrollo de actividades } \\
\text { educativas. } \\
\text { - Comunica con claridad } \\
\text { ideas, tomando en cuenta } \\
\text { las contribuciones de los } \\
\text { demás usuarios. } \\
\text { - Intercambia } \\
\text { experiencias en el } \\
\text { mundo virtual }\end{array}$ & $\begin{array}{l}\text { - ¿Cuáles son las características de espacios } \\
\text { propicios para adecuadas experiencias de } \\
\text { aprendizaje? } \\
\text { - Dentro de SL existe todo tipo de lugares y } \\
\text { cada cual es una experiencia distinta. } \\
\text { ¿Cómo proponer límites a los estudiantes } \\
\text { para que no se dirija a lugares no } \\
\text { beneficiosos? } \\
\text { - ¿Considera que el intercambio de } \\
\text { experiencias en SL fomenta el aprendizaje } \\
\text { en este tipo de entornos? }\end{array}$ \\
\hline & $\begin{array}{l}\text { Cooperación } \\
\text { e interacción } \\
\text { en el } \\
\text { aprendizaje }\end{array}$ & $\begin{array}{l}\text { - Organiza y distribuye } \\
\text { tareas entre } \\
\text { participantes, respetando } \\
\text { sus roles. } \\
\text { - Evalúa el trabajo entre } \\
\text { pares, teniendo en cuenta }\end{array}$ & $\begin{array}{l}\text { - En los trabajos en equipo. ¿Cómo se } \\
\text { distribuyen las actividades entre los } \\
\text { integrantes? } \\
\text { - Cuando se realizan trabajos en equipo. } \\
\text { ¿Cómo se evalúa el trabajo de cada } \\
\text { integrante? }\end{array}$ \\
\hline
\end{tabular}


Realizada la ruta metodológica, permitió realizar las observaciones en la institución educativa superior, donde las secuencias de acciones fueron realizadas por docentes con habilidades tecnológicas, junto a sus estudiantes, donde el objetivo fue lograr experiencias de aprendizaje inmersivo a través de recursos tecnológicos a través de la plataforma Second Life, donde los estudiantes establecieron conexión a través de sus computadoras. Asimismo, se realizaron entrevistas a profundidad a expertos en entornos virtuales y con la ayuda del atlas ti, se pudo establecer que esta plataforma permite la interacción con diversas personas, inclusive con estudiantes de otros países, de culturas distintas, donde el aprendizaje es de alto nivel.

En la sesión se observó el saludo del docente a sus estudiantes, donde realiza preguntas respecto a la temática de la sesión: por ejemplo: ¿Conocen algunos lugares del Perú? ¿Qué es lo que recuerdas de algún viaje que hayas tenido? ¿Tienes fotos de tus viajes? ¿Por qué las personas toman fotos en los viajes? donde las respuestas fueron añadidas en la proyección multimedia dirigida a los estudiantes.

Es así como el aprendizaje inmersivo experiencial presenta diversas actividades que ha permitido que los estudiantes se sumerjan en un lugar que se asemeja a la realidad y que es construido de manera artificial (mundo virtual). En Second Life he desarrollado proyectos en la construcción de espacios, objetos, realización de animaciones, entre otras cosas, así también la enseñanza y aprendizaje para la realización de estas actividades dentro de espacios virtuales. "Puede ser Egipto, la antigua Roma, Grecia, obtener un panorama futurista, con Second Life puedo llegar a ellos. Cada lugar maneja un vocabulario definido es por ello que, el interactuar con participantes dentro de este 
entorno ayuda a tener experiencias de distinto índole y aprender de ellas" afirmó la Dra. Eugenia Calderón (México).

En la sesión, los estudiantes se conectaron través de sus equipos e iniciar sesión accediendo al mundo virtual, a través de sus avatares, que son unas representaciones gráficas tridimensionales de cada uno de los estudiantes. Adicional a ello, el docente mostró el uso de las herramientas necesarias para la interacción dentro de este mundo virtual, herramientas tales como: búsqueda de lugares, avanzar, volar, saltar, tomar fotografías, teleportar, uso de notecard, uso del chat, asimismo, herramientas para configuración de apariencia del avatar. La docente menciona que estas herramientas son necesarias para propiciar una buena interacción entre los estudiantes y los lugares que visita.

Para Germán Ariel (Argentina), el estudiante "debe probar, equivocarse, sorprenderse, remangarse, bajar a la arena. En una palabra, implicarse y, por supuesto divertirse haciéndolo, entretenerse mientras actúa; el juego y las formas de trabajo participativo que permiten hacer son esenciales en situaciones de aprendizaje"

El Second Life permite la posibilidad de crear experiencias y desarrollar nuestros propios juegos de inmersión, lugares de música, clubes de baile, espacios de entretenimiento para adultos o cualquier experiencia virtual que se pueda imaginar. "he realizado actividades de observación e investigación en clases formales en el ambiente inmersivo, en la Asignatura Dibujo Geométrico. También aprovechar los lugares y las construcciones que existen en SL para poder explicar de una manera más gráfica y motivante, y que los participantes puedan a través de la imaginación y el descubrimiento, maravillarse y aprender significativamente."

Haciendo uso de las herramientas teleport, el docente invitó a cada uno de los estudiantes a visitar diversos lugares del mundo virtual Second Life; asimismo, indicó a los estudiantes a describir a través del chat los lugares que observa teniendo en cuenta la buena redacción; visitando la "Réplica de Caral", el cual ha sido diseñado y construido por la docente. Visitaron toda la extensión del terreno que tiene un aproximado de $2300 \mathrm{~m}^{2}$ y tiene una zona destinada como patrimonio cultural, en el cual se ubica la ciudadela de Caral con cada uno de sus espacios y lugares ceremoniales, los cuales, al tocarlos, muestran a través de una animación, la información real de esta cultura peruana. Las construcciones básicas son para utilizarlas como apoyo pedagógico o como experiencia de construcción y programación.

Los estudiantes interactuaron en los espacios de Caral, llamando la atención las pirámides, las cuales han sido diseñadas a escala y sobre todo la pirámide de anfiteatro en la cual hay distintos tipos de animaciones que nos invita a informarnos sobre la construcción de este lugar. Dentro de este espacio hay un panel virtual que nos muestra en resumen de manera gráfica los objetos encontrados dentro de este espacio. Los estudiantes al sumergirse dentro de este espacio evidencian su asombro por la forma como fue diseñado este espacio. Otros mostraron el deseo de visitar Caral de manera presencial.

Para Limberg Suñe (Chiclayo- Perú) es importante que el docente tenga en cuenta conocer en qué nivel se encuentra cada usuario que está participando en este mundo virtual, estar familiarizado con su avatar y de esa forma iniciar con construcciones sencillas en dos dimensiones y luego realizar construcciones más complejas, como rediseñar espacios propuestos en el entorno.

En este terreno se observa una zona denominada como patrimonio natural, el cual nos muestra las riquezas en flora y fauna del Perú, con animales oriundos de nuestro país, tales como el gallito de las rocas, el otorongo, entre otros ejemplares. Los estudiantes al dirigirse a este espacio interactuaron 
con los animales que están en este espacio, obtienen información de animales y plantas que se encuentra en este terreno, los estudiantes intercambiaron opiniones respecto la importancia de preservar estos ambientes y que la mano humana debería mantenerse alejada de estos espacios para que la naturaleza pueda abrirse camino frente a las distintas formas de vida que existe en lugares como este.

Desde mi punto de vista los espacios que se pueden construir en Second Life deben tener las mismas características que un espacio presencial, por ejemplo, si voy a realizar un trabajo de laboratorio en temas ambientales el espacio virtual debe ser de igual características o mejores que un laboratorio. Limberg Suñe (Chiclayo- Perú).

Adicionalmente, la docente muestra e invita a los estudiantes a realizar algunas pequeñas construcciones dentro de este mismo espacio, permitiendo a los estudiantes desenvolverse diseñando espacios y teniendo noción de las dimensiones que puede tener algún objeto que puedan construir, de la misma forma, asignar a cada objeto la animación respectiva, con la finalidad que cada visitante pueda interactuar con el objeto construido.

Para Susana Varsavsky (Argentina), las características del objeto dependen de lo que se quiere enseñar con él, pero podría acotar, como cosa general, que lo que mayormente se busca es que el objeto en cuestión y su forma de manipularlo sea lo más parecido a la realidad para que la simulación transmita la idea más cercana a lo que sucede en la realidad.

Se pidió a los estudiantes que en equipos realicen un texto descriptivo y argumentativo en drive, en el cual consignen las diferencias que evidencian entre un patrimonio cultural y un patrimonio natural, asimismo, puedan argumentar por qué estos dos tipos de patrimonios deben ser protegidos por la sociedad y si es necesaria la intervención de la mano del hombre para que estos espacios se sustenten por sí mismos.

Es importante que el docente marque una ruta definida para que el aprendizaje dentro de este mundo virtual sea lo más beneficioso posible y que las experiencias que pueda asimilar puedan enriquecer su aprendizaje, así también el estudiante podrá entender que las clases no son improvisadas, sino que hay un diseño previo y objetivos que se deben cumplir en la construcción del aprendizaje.

Al finalizar la sesión se observó que los estudiantes estaban muy motivados, deseosos de continuar con su avatar y seguir sus clases, descubriendo nuevas cosas, recreando otras, interactuando entre ellos, cuyos aprendizajes quedaron demostrados con sus actividades realizadas y las evidencias mostradas de su aprendizaje.

Hay que tener en cuenta que una clase en Second Life es una clase que simula un entorno real, por tal razón los indicadores deben estar alineados a este aspecto, así también, al fomento de la responsabilidad, la disciplina y la atención de tal forma que el aprendizaje no se quede en aspectos secundarios sino que se cumplan los objetivos que se trazan al comienzo; un aspecto importante también es que los participantes puedan estar contentos y satisfechos de lo que se realiza en clase, esto se demuestra cuando están deseosos de seguir en los cursos por más que estos sean de naturaleza libre. Es decir, los medios utilizados nos han permitido lograr aprendizajes significativos, con estudiantes motivados y con el deseo de continuar su proceso.

Por ello, para que este entorno sea beneficioso en la enseñanza aprendizaje, siempre que el maestro junto con los estudiantes pueda realizar una inducción respecto los beneficios y los peligros que pueden existir al desenvolverse en los mundos virtuales. Asimismo, cada participante debe 
conocer las ventajas y que estas motiven para saber que se puede aprender y sacar el mayor provecho posible de los lugares que se visitan y que son potenciales lugares para aprender.

Por otro lado, también permite el desarrollo de habilidades blandas, como el trabajo en equipo, donde se pueden presentar diferentes variantes; ejemplo si los participantes son de diferentes especialidades como en muchos casos ha pasado, uno debe aportar lo que mejor sabe hacer, algunos realizan el proceso de construcción otros el proceso de organización otros pueden hacer presentaciones, es decir, hay un trabajo mancomunado pero cada uno por todo desde su especialidad.

La experiencia realizada por los docentes demuestra la efectividad de los aprendizajes, tanto de las habilidades duras como blandas, desarrollando y fortaleciendo las competencias y con el gran apoyo de las herramientas tecnológicas. Queda demostrado también, que este logro depende de los dos actores más importantes en el proceso de aprendizaje: el docente y el estudiante, que permite a ambos seguir aprendiendo, como producto de la interacción entre ambos, utilizando los avatares.

\section{Discusión}

El proceso de aprendizaje a través del Second Life ha demostrado que el aprendizaje realizado por los estudiantes universitarios ha sido efectivo, con el desarrollo de las competencias preestablecidas. Esto es reafirmado por diversos investigadores donde afirman que los estudiantes al sumergirse en estas actividades logran aprendizajes significativos (Ly, Saadé \& Morin, 2017).

Así también está conformado por actividades sumergiéndose es el mundo virtual interactuando con "personas" objetos logrando aprendizajes que sería muy complicado en la realidad y por lo tanto el aprendizaje es efectivo (Inks y Ávila, 2008). Además, también queda demostrado que la binomio docente-estudiante es fundamental para seguir aprendiendo ambos sujetos, con una interacción dinámica, jugando un papel los "avatares" para ello. Esto lo confirma Cheney y Terry (2018) cuando afirma que los estudiantes se presentan, se encarnan en personajes y logran un aprendizaje "transformacional".

Cuando se inicio las sesiones de aprendizaje, no se dejo de percibir los procesos de la gestión de aula, desde el saludo cordial entre docente-estudiante y la motivación, que de por si es permanente por las actividades que realizan desarrollando las competencias y capacidades programadas. Por ello este proceso es una motivación (intrínseca-extrínseca) para los estudiantes (Abbott, Jeffrey, Gouseti, Burden, \& Maxwell, 2017).

Los aprendizajes inmersivos requieren una preparación por parte del docente, donde los escenarios de aprendizaje (de acuerdo a los objetivos y competencias programadas) sean lo mas cercano a la realidad de estudio (mundo virtual). Esto permite centrar el aprendizaje en el problema a resolver, desarrollando que el aprendizaje procedimental y pensamiento complejo sea enriquecido a través del juego (Mystakidis \& Berki, 2018; Kim, Song, Lockee \& Burton, 2018).

Cuando los estudiantes lograron interactuar a través de sus avatares, a través de herramientas otorgadas por el docente, se observó la motivación, creatividad, la comunicación y el trabajo en equipo en todos los participantes, logrando e impactando en los aprendizajes, donde comprenden que aprender el día de hoy es diferente en la actualidad. Por ello, la simulación (realidad virtual) es una forma de lograr una auténtica oportunidad de aprendizaje (Ly, Saadé \& Morin, 2017; Beckem \& Watkins, 2012).

El Second Life ha permitido recrear experiencias educativas a través del juego creando islas de aprendizajes como de recreación. Esto debe estar vinculado a que el docente esté preparado para 
adaptar los enfoques educativos para lograr aprendizajes, sin descuidar el rigor epistemológico, el lenguaje académico y el entorno social (Nuñez \& Duarte, 2017; Hand, Cavagnetto, Chen \& Park, 2016).

Esto fue observado claramente cuando visitaron la replica de Caral, donde los estudiantes se mostraron fascinados, motivados por lo estaban realizado. Es por eso, este nuevo escenario de aprendizaje viene desarrollando nuevas formas de aprender, compartiendo los nuevos conocimientos y trabajando en equipo (Ángel, Valdés y Guzmán, 2017; Quinche y González, 2011; Márquez, 2010).

El proceso de la evaluación de los aprendizajes realizado ha permitido al docente tener un acercamiento real al logro establecido tanto en competencias cognitivas, procedimentales y actitudinales de los estudiantes, debido a la variedad de indicadores de aprendizajes que se realizan en una inmersión de aprendizaje. Por ello, los mundos virtuales se convierten en un lugar ideal para realizar actividades de aprendizaje por medio de las distintas representaciones de la realidad (Cruz, Therón, Pizarro y García-Peñalvo, 2013).

Entre las principales conclusiones podemos afirmar que el aprendizaje inmersivo experiencial, ha sido exitoso en la medida que los actores educativos directos han utilizado un conjunto de actividades en el entorno virtual logrando aprendizajes significativos, asimismo lograron integrarse con las diversas herramientas diseñando, construyendo y evaluando los espacios creados para su aprendizaje.

Con referencia a aprendizaje inmersivo constructivista los docentes realizaron procesos de planificación de los aprendizajes, generando la reflexión por parte de él y los estudiantes de las islas creadas, comunicándose activamente los diversos actores intercambiándose experiencias de aprendizaje a través de trabajo en equipo y generando una evaluación entre pares permanente. Con el aprendizaje inmersivo social o colaborativo generaron que el docente y estudiante realicen propuestas de aprendizaje a través de proyectos y actividades, generando una mayor autonomía en su proceso de aprendizaje. Asimismo, han generado una comunicación activa, compartiendo experiencias adecuando diversas experiencias o modelos propuestos.

Por tanto, en torno a estas tres categorías se afirma que el aprendizaje inmersivo se convierte en una alternativa para el aprendizaje en las diversas áreas de aprendizaje, donde es elemental que los docentes se involucren en las tecnologías como herramienta para el aprendizaje y que las instituciones educativas actualicen su tecnología para la implementación

\section{Referencias}

Abbott, D., Jeffrey, S., Gouseti, A., Burden, K., \& Maxwell, M. (2017). Development of crosscurricular key skills using a 3D immersive learning environment in schools. Communications in Computer and Information Science, 725, 60-74. doi: https://doi.org/10.1007/978-3-31960633-0_6

Ángel, C., Valdés, J., \& Guzmán, T. (2017). Límites, desafíos y oportunidades para enseñar en los mundos virtuales. Journal Educational Innovation / Revista Innovación Educativa, 17(75), $149-168$.

Beckem, J., \& Watkins, M. (2012). Bringing Life to Learning: Immersive Experiential Learning Simulations for Online and Blended Courses. Journal of Asynchronous Learning Networks, 16(5), 61-70. doi: https://doi.org/10.24059/olj.v16i5.287

Buitrago, B. (2016). Mundos virtuales 3d orientados a la enseñanza. Journal Boliviano de Ciencias, 12(37), 34-39. 
Bulkley, K. (11 de mayo de 2007). Entrevista con Philip Rosedale. Recuperado de https://goo.gl/HtEPKo

Cheney, A., \& Terry, K. (2018). Immersive Learning Environments as Complex Dynamic Systems. International Journal of Teaching \& Learning in Higher Education, 30(2), 277-289.

Cruz, J., Therón, R., Pizarro, E., \& García-Peñalvo, F. (2013). Knowledge Discovery in Virtual Worlds Usage Data: approaching Web Mining concepts to 3D Virtual Environments. In Complementary proceedings of the The Fourth International Workshop on Knowledge Discovery, Knowledge Management and Decision Support - Eureka-2013, Mazatlán, México.

Diaz, S. (2016). Mundos virtuales. Metaanálisis de experiencias educativas desde sus inicios. EnseñAnza \& Teaching, 34(2), 43-63. doi: https://doi.org/10.14201/et20163424363

Elías, J., \& Evaristo, I. (2009). Reflections on Academic use of the Second Life environment in higher education. Recuperado de https://goo.gl/txykQG

Fernandes, K., Raja, V., \& Eyre, J. (2003). Immersive learning system for manufacturing industries. Computers in Industry, 51(1), 31-40. doi: https://doi.org/10.1016/S0166-3615(03)00027-7

Gabarda, S., Orellana, N., \& Pérez, A. (2017). La comunicación adolescente en el mundo virtual: una experiencia de investigación educativa. Revista de Investigación Educativa, 35(1), 251-267. doi: http://dx.doi.org/10.6018/rie.35.1.251171

García-Peñalvo, F., Cruz-Benito, J., \& Therón, R. (2014). Visualización y Análisis de Datos en Mundos Virtuales Educativos: Comprendiendo la interacción de los usuarios en los entornos 3D. ReVisión, 7(2), 46-59.

Grané, M., Frigola, J. y Muras, M. (2007). Second Life: Avatares para aprender. Recuperado de http://161.116.23.180/repositori/objectes/mg0001/sl_aprender.pdf

Hand, B., Cavagnetto, A., Chen, Y., \& Park, S. (2016). Moving Past Curricula and Strategies: Language and the Development of Adaptive Pedagogy for Immersive Learning Environments. Research in Science Education, 46(2), 223-241. doi: https://doi.org/10.1007/s11165-0159499-1

Hernández, R., Fernández, C., \& Baptista, P. (2014). Metodología de la investigación. (6º ed.). México: Educación

Inks, S., \& Avila, R. (2008). Preparing the Next Generation of Sales Professionals Through Social, Experiential, and Immersive Learning Experiences. Journal of Advancement of Marketing Education, 13, 47-55.

Joyanes, L. (2017). Industria 4.0 La cuarta revolución industrial. España: Marcombo.

Kim, S., Song, K., Lockee, B., \& Burton, J. (2018). Gamification in Learning and Education. doi: https://doi.org/10.1007/978-3-319-47283-6

Ly, S., Saadé, R., \& Morin, D. (2017). Immersive learning: Using a web-based learning tool in a phd course to enhance the learning experience. Journal of Information Technology Education Research, 16, 227-246. doi: https://doi.org/10.28945/3732

Martinez, R. (2014). Sloodle. Conexión de entornos de aprendizaje. Barcelona, España: Editorial UOC.

Marquez, I. (2010). La simulación como aprendizaje: educación y mundos virtuales. Universidad Complutense de Madrid, España.

Mystakidis, S., \& Berki, E. (2018). The Case of Literacy Motivation: Playful 3D Immersive Learning Environments and Problem-Focused Education for Blended Digital Storytelling. International Journal of Web-Based Learning and Teaching Technologies. 13(1). doi: https://doi.org/10.4018/IJWLTT.2018010105

Negroponte, N. (1995). El mundo digital. Barcelona, España: Ediciones B. ISBN 84-406-5925-3

North, M. (2014). Distance learning immersive environments: Sense of presence exploration. Journal of Visual Languages \& Computing, October. doi: https://doi.org/10.1016/j.jvlc.2014.09.006

Nuñez, L., \& Duarte, A. (2017). Posibilidades educativas de un mundo virtual 3D (Tesis Doctoral). Universidad de Huelva, España.

Propósitos y Representaciones

Ene. - Abr. 2020, Vol. 8, $\mathrm{N}^{\circ} 1$, e430

http://dx.doi.org/10.20511/pyr2020.v8n1.430 
Núñez, L., Giordano, M., Menacho, I., \& Uribe, Y. (2019) Competencias administrativas en la calidad del servicio en las instituciones educativas del nivel inicial. Apuntes Universitarios, 9(1). https://doi.org/10.17162/au.v1i1.347

Núñez, L., Bravo, L., Cruz, C., \& Hinostroza, M. (2018) Competencias gerenciales y competencias profesionales en la gestión presupuestaria. Revista Venezolana de Gerencia, 23(83), 2018, 761-778.

Olbrish, K. (2013). Immersive Learning: Designing for Authentic Practice. Estados Unidos:American Society for Training \& Development.

Paz, P. (2017). Los mundos virtuales, estructura y manifestación, el caso de WoW: La creación del sujeto y la transposición lingüística (Tesis de pregrado). Pontificia Universidad Católica del Ecuador, Ecuador.

Popper, K. (1980) La lógica de la investigación científica. Traducción de Víctor Sánchez de Zavala. (5ta reimpresión) Madrid, Tecnos.

Quinche, J., \& González, F. (2011). Entornos Virtuales 3D, alternativa pedagógica para el fomento del aprendizaje colaborativo y gestión del conocimiento en Uniminuto. Formación Universitaria, 4(2), 45-54. doi: http://dx.doi.org/10.4067/S0718-50062011000200006

Stefan, L. (2012). Immersive collaborative environments for teaching and learning traditional design. Procedia - Social and Behavioral Sciences, 51, 1056-1060. doi: https://doi.org/10.1016/j.sbspro.2012.08.287

Yuni, J., \& Urbano, C. (2005) Mapas y herramientas para conocer la escuela, investigación etnográfica e investigación-acción. ( $3^{\circ}$ ed.) Córdoba: Brujas. 